\title{
ETC Observations of the Gamma-ray Burst GRB 941014
}

\author{
Roland Vanderspek, George R. Ricker \\ Center for Space Research, Massachusetts Institute of Technology, Cambridge, USA
}

\begin{abstract}
The Explosive Transient Camera $(E T C)$ is a dedicated wide-field sky monitor for short-duration optical transients. The ETC operates completely automatically, searching for transients in the night sky without the need for a human observer on site. Data products from the ETC include data from any transients detected, as well as CCD images of the fields-of-view observed. We report here the imaging by the $E T C$ of the $2.5 \sigma$ error region of GRB 941014 90 seconds after the end of the burst.
\end{abstract}

\section{Introduction}

The Explosive Transient Camera $(E T C)$ is an automated, wide-field sky monitor instrument designed to detect short-timescale optical transients in real time. The ETC was developed to detect transient optical radiation associated with $\gamma$-ray bursts (GRBs); however, with its large field-of-view and high sensitivity, the $E T C$ is able to detect other, perhaps previously unknown, classes of fast celestial optical transients.

The $E T C$ has been operating fully automatically from the summit ridge of Kitt Peak (USA) since 1990. Since then, the ETC has monitored over 1000 steradian-hours of the night sky in the search for one-second optical transients as faint as $\mathrm{V} \approx 10$. The $E T C$ has also collected and stored over 35000 wide-field images of the night sky to limiting magnitudes ranging from $\mathrm{V} \approx 11-13$.

Since the launch of the Compton Gamma Ray Observatory (CGRO), the data collected from $E T C$ observations have been compared with the locations of GRBs detected by the Burst and Transient Source Experiment (BATSE) on $C G R O$. If a GRB detected by BATSE is localized to have been in the ETC field-of-view during $E T C$ observations and no optical radiation was detected, an upper limit on the ratio of the GRB fluence to the fluence of prompt optical radiation $\left(S_{\gamma} / S_{\text {opt }}\right)$ can be set. To date, five such coincidences have occurred, with no correlated optical radiation (Krimm et al. 1994): the upper limits of $S_{\gamma} / S_{\text {opt }}$ calculated for these bursts range from 2-100. However, the error regions of these GRBs are so large (typically $4^{\circ}-8^{\circ}$ radius $(1 \sigma)$ ), that even the large ETC field-of-view cannot cover the $2 \sigma$ error region, so only partial spatial coverage of these bursts was possible. 


\section{The Explosive Transient Camera}

\subsection{Instrumentation}

The Explosive Transient Camera $(E T C)$ consists of a bank of sixteen wide-field CCD cameras: each camera has a field-of-view of $20^{\circ} \times 15^{\circ}$. The ETC fieldsof-view overlap in pairs to allow anticoincidence techniques to be used to reject local sources of optical transients: the overall field-of-view of the ETC system is 0.73 steradians. The characteristics of the $E T C$ are given in Table 1 ; further details can be found in Vanderspek et al. (1992).

Table 1. The optical characteristics of the Explosive Transient Camera

\begin{tabular}{lc}
\hline Characteristic & Value \\
\hline Location & Kitt Peak, USA \\
Number of Cameras & 16 \\
Optical System & $\mathrm{CCD}+24 \mathrm{~mm}$ lens \\
Field-of-View per Camera & $20^{\circ} \times 15^{\circ}$ \\
Pixel Angular Size & 3.1 arc-minutes \\
Total Field-of-View & 0.73 steradian \\
Bandpass & $4000 \mathrm{~A}-7500 \mathrm{~A}$ \\
Limiting magnitude (field stars) & $\mathrm{V} \cong 10.5(4 \sigma, 5 \mathrm{~s}$ exposure) \\
Limiting magnitude (1s flash) & $\mathrm{V} \approx 8-10(10 \sigma)$ \\
\hline
\end{tabular}

\subsection{Operations}

The $E T C$ was designed as an automatic instrument, to reduce the time and cost of having a human operator on site. The ETC control software has complete control of the ETC instrumentation (including the roll-off roof) and, through peripheral hardware, is aware of the local weather and sky conditions. The software has been designed to "think" as a human observer would, and so can operate with the same high efficiency a human observer would.

$E T C$ observations consist of a continuous, contiguous series of short (5s) exposures of the night sky by all cameras simultaneously. Each image is compared, pixel-by-pixel, to the one taken immediately preceding, to find pixels which have brightened by a significant amount. If a brightening is seen at the same celestial coordinates by two ETC cameras in the same exposure, they are considered images of a real celestial optical transient and data from the event are stored.

At regular intervals, theETC stores full images of the night sky from all CCD cameras. These images are used to check instrument health, and are later analyzed to look for long-term variability in field stars.

The $E T C$ is also capable of responding to alerts from the $B A C O D I N E$ system (Barthelmy et al. 1994). When a GRB alert is received by the ETC system while the $E T C$ is observing, the cameras are slewed to the location of the burst, and images of the burst field are taken and stored. 


\section{Observations of GRB 941014}

On 14 October, 1994, BATSE detected a bright GRB: the fluence is estimated to be $10^{-5} \mathrm{erg} \mathrm{cm} \mathrm{cm}^{-2}$ in the $57-318 \mathrm{keV}$ energy band (C. Kouveliotou, private communication). The BACODINE system intercepted the BATSE telemetry stream, calculated the burst coordinates, and distributed them to its member sites, and electronic mail describing the burst was sent to MIT the following morning. The mail message described a burst which occurred in the field-ofview of the $E T C$ cameras at a time when the $E T C$ was observing: it turned out that the ETC was storing data to disk at the time of the BACODINE alert, and was, therefore, insensitive to the burst alert. However, the ETC restarted its observations soon thereafter and stored full images of the sky from all 16 CCD cameras. Analysis of the light curve of this burst (C. Kouveliotou, private communication) showed that these images were taken 90 seconds after the end of the burst.

Because the localization precision of $B A C O D I N E$ ( $8^{\circ}$ radius $(1 \sigma)$ ) is so large, the $E T C$ images covered only a fraction of the error region calculated by $B A$ CODINE. When the Interplanetary Network (IPN) arc was made available (K. Hurley, private communication), the error region was reduced to the intersection of a narrow $\left(0^{\circ} 25\right)$ arc and the large $B A C O D I N E$ error circle: this arc was partially imaged by one pair of ETC cameras. When the more precise $B A T S E$ error region was made available (G. Fishman, private communication), it turned out that the entire $2.5 \sigma$ error region of this bright GRB had been imaged by a pair of $E T C$ cameras. This image and the $2 \sigma B A T S E / I P N$ error region are shown in Fig. 1. Because the center of the GRB error region is off-center in the $E T C$ image, the coverage of the error region is asymmetric: the image contains the error region to $2.5 \sigma$ on one side and to $5.5 \sigma$ to the other.

\section{Results and conclusions}

The analysis of the ETC image of the $2.5 \sigma$ error region of GRB 941014 revealed that the limiting magnitude of the image was $V=10.2(4 \sigma)$, and that four stars were within the error region. Analysis of an image taken of the same field taken two nights earlier revealed the same four stars. Thus, no new optical radiation was detected in the $2 \sigma$ error region of GRB 941014. The absence of optical radiation corresponds to an upper limit on the ratio of the optical ( $V$ band) fluence 90 seconds after the end of the burst to the $\gamma$-ray burst fluence (57-318 keV; C. Kouveliotou, private communication) of $1 \cdot 10^{-4}$.

Acknowledgements: We would like to thank the BATSE team for their assistance in getting the error region and light curve for GRB 941014: specifically, Gerald Fishman and Chryssa Kouveliotou were very quick in their responses to our queries. We would also like to thank Kevin Hurley for his assistance in getting the IPN coordinates. Scott Barthelmy was very quick and gracious in his relaying of BATSE and IPN information, in part via the BACODINE network. The ETC is supported under grants NAGW-2089 and NAG5-2165. 


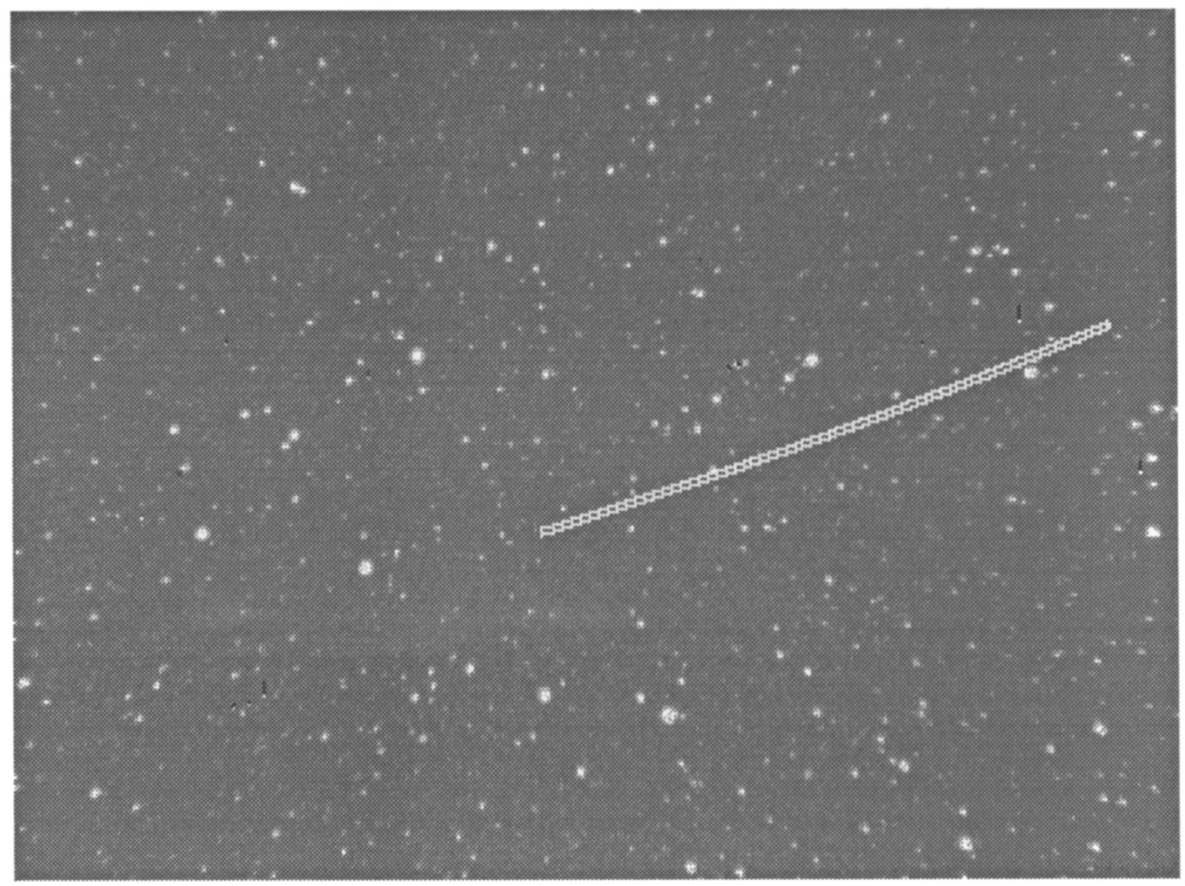

Fig. 1. ETC image of the error region of GRB 941014: the image measures $20^{\circ} \times 15^{\circ}$, with a pixel size of $3 ! 1$. Stars as faint as $\mathrm{V}=10.5$ are visible on this image. The thin arc encloses the error region of GRB 941014: the dimensions of the arc are $10^{\circ}$ long $(2 \sigma$ error, as defined by BATSE) by $80^{\prime \prime}$ wide ( $3 \sigma$ error, as defined by the Interplanetary Network). In this image, the thickness of the arc is exaggerated for clarity: the actual $3 \sigma$ arc thickness is only 0.4 pixels.

\section{References}

Barthelmy S.D., et al., 1994, in Gamma-Ray Bursts, G.J. Fishman, J.J. Brainerd, K. Hurley (eds.), AIP Conf. Proc. 307, AIP, New York, p. 643

Krimm H.A., Vanderspek R., Ricker G.R., 1994 in Gamma-Ray Bursts, G.J. Fishman, J.J. Brainerd, K. Hurley (eds.), AIP Conf. Proc. 307, AIP, New York, p. 423

1 Vanderspek R., Ricker G.R., Doty J.P., 1992 in "Robotic Telescopes in the 1990s, A. V. Filippenko (ed.), Astronomical Society of the Pacific Conf. Proc. 34, A.S.P., San Francisco, p. 123 